Sharif University of Technology
Scientia Iranica
SCIENTIA

\title{
Effects of hypertension on Intima-Media Thickness (IMT); application to a human carotid artery
}

\author{
S.A. Mirbagheri, M.S. Saidi*, S. Sohrabi, B. Firoozabadi and M.H. Banazadeh \\ School of Mechanical Engineering, Sharif University of Technology, Tehran, Iran.
}

Received 27 July 2014; received in revised form 31 July 2015; accepted 16 January 2016

KEYWORDS

Atherosclerosis;

Mass transport;

LDL;

CFD;

Intima;

Carotid bifurcation.

\begin{abstract}
A vast number of deaths in the world have been attributed to atherosclerosis. The prominent aim of this study is proposing an accurate and simple model to investigate the process of arterial wall thickening. In order to investigate LDL (Low Density Lipoprotein) accumulation in arterial wall, a four layer model for arterial wall consisting of endothelium, intima, IEL, and media is presented. All layers are treated as homogenous porous media. This model has been solved both numerically and analytically. Obtained accumulated LDL in the intima is used to calculate oxidized LDL flux. Also, the presented model and clinical data are used to prepare the growth model for arterial wall. Furthermore, the effect of hypertension on filtration velocity and rate of wall thickening has been studied which is in consistent with experimental data. Results show that the average rates of intima thickening of hypertensive patients with $120 \mathrm{mmHg}$ and $160 \mathrm{mmHg}$ transmural pressure are $5.87 \mu \mathrm{m} /$ year and $6.12 \mu \mathrm{m} /$ year, respectively. This rate for healthy subjects with $70 \mathrm{mmHg}$ transmural pressure has been calculated $4.5 \mu \mathrm{m} /$ year. Finally, this model is applied to a carotid artery, and the maximum intimal growth rate for people with $70 \mathrm{mmHg}$, $120 \mathrm{mmHg}$ and $160 \mathrm{mmHg}$ transmural pressure is calculated $4.68 \mu \mathrm{m} /$ year, $6.28 \mu \mathrm{m} /$ year, and $6.67 \mu \mathrm{m} /$ year, respectively.

(C) 2016 Sharif University of Technology. All rights reserved.
\end{abstract}

\section{Introduction}

Atherosclerosis, as one of the prevalent cardiovascular diseases (CVD) [1], is characterized by accumulation of Low Density Lipoprotein (LDL) in arterial wall [2]. It commonly develops in large- and medium-sized elastic arteries [3]. The transport process of atherogenic species, which is called arterial mass transport such as LDL, from the bulk blood flow in lumen to arterial wall, makes a significant contribution to lipid accumulation in arterial wall [4]. The LDL accumulation, resulted

*. Corresponding author. Tel.: +98216616 5558; Fax: +982166000021

E-mail addresses: mirbagheri@alum.sharif.edu (S.A.

Mirbagheri); mssaidi@sharif.edu (M.S. Saidi);

sohrabi@mech.sharif.edu (S. Sohrabi);

firoozabadi@sharif.edu (B.Firoozabadi);

mhbanazadeh@alum.sharif.edu (M.H.Banazadeh) from a progressive disorder in arterial wall, leads to gradual narrowing of arteries and plaque formation [1].

It is concurred that atherosclerosis lesions do not occur in a random fashion [5]. Today, researchers agree that atheroma develops in regions with complex flow pattern. Sites of Branching, bifurcation, bending, and local dilation are examples of these regions [6-8].

Another critical event for development of atherosclerosis are inflammatory cells, including monocytes, macrophages, and lymphocytes (predominantly $\mathrm{T}$ cells) [5]. Early atherosclerotic lesion is a common site for accumulation of monocytes and $\mathrm{T}$ lymphocytes. Ingesting oxidized LDL ( $\left.\mathrm{LDL}_{\mathrm{ox}}\right)$, macrophages turn into monocytes in the artery wall and entirely become foam cells. Although there is a complex process for plaque formation, some researchers believe that it is initiated by the oxidation of accumulated LDL [5]. The first step to model abnormal enlargement 
of intima is modeling LDL accumulation in arterial wall.

The main focus of the present study is developing a new, simple and accurate model for intimal thickening in order that it can be easily applied to complex geometries such as carotid artery. Accumulated LDL in the intima is the first stage of arterial wall thickening. A homogenous reliable four-layer model is used to investigate LDL concentration in the intima, both analytically and numerically. Applying analytical results to growth model, which is based on oxidation of LDL, we developed a practical, however, simple model to predict IMT (Intima-Media Thickness) for healthy subjects and more interestingly for hypertensive cases. These results agree well with clinical and experimental data. Finally, the model is applied to a carotid artery in order to investigate LDL accumulation in the carotid arterial wall and predict the maximum intimal growth rate of the artery.

\section{Mathematics formulation}

The arterial wall consists of endothelium, intima, IEL and media which are all considered macroscopically homogenous porous media. The detailed description of the model is presented by Yang and Vafai [9].

\subsection{Lumen}

Flow in the lumen is assumed to be incompressible and Newtonian, so the governing equations can be expressed as:

$$
\begin{aligned}
& \rho V . \nabla V-\mu \nabla^{2} V+\nabla p=0, \\
& \nabla \cdot V=0,
\end{aligned}
$$

where $V, p, \rho$, and $\mu$ are the velocity vector, pressure, density, and dynamic viscosity of blood, respectively.

Via the mass transport equation, concentration field is obtained by:

$$
V . \nabla c=D \nabla^{2} c
$$

where $c$ is the LDL concentration and $D$ is the LDL diffusivity in the blood.

\subsection{Arterial wall}

Endothelium, internal elastic lamina, intima, and media are treated as macroscopically homogenous layers and modeled as:

$$
\begin{aligned}
& \frac{\mu}{K}\langle V\rangle=-\nabla\langle p\rangle+\mu^{\prime} \nabla^{2}\langle V\rangle, \\
& \nabla \cdot\langle V\rangle=0, \\
& \left(1-\sigma_{f}\right)\langle V\rangle \cdot \nabla\langle c\rangle=D \nabla^{2}\langle c\rangle+k\langle c\rangle,
\end{aligned}
$$

where $K$ is the hydraulic permeability, $\mu$ the effective dynamic viscosity, which is chosen as $\mu / \varepsilon, \varepsilon$ the porosity, $k$ the effective volumetric first-order reaction rate, and $\sigma_{f}$ the Staverman filtration osmotic reflection coefficient. The symbol \langle\rangle shows the local volume average of a quantity. It should be noted that reaction only happens in the media, so $k$ is equal to zero for other layers.

\subsection{Growth model for intima}

Using Eqs. (1)-(6) and changing intima thickness, we calculated the concentration in intima as a function of this layer thickness. Average dimensionless LDL concentration in intima and accumulated mass per unit area are defined as:

$$
\begin{aligned}
& C_{\mathrm{avg}}=\frac{\int_{0}^{\varepsilon} c d r}{\varepsilon}, \\
& m=c_{0} \int_{0}^{\varepsilon} \varepsilon C d r,
\end{aligned}
$$

where $\varepsilon$ is the intima thickness.

The production of oxidized LDL is because of LDL oxidation by reaction with the radicals and a reduction of oxidized LDL through ingestion by macrophages [10]. Based on the assumption that the ingestion process does not change the concentration of oxidized LDL noticeably, the production of oxidized LDL is modeled as:

$$
\frac{d c_{\mathrm{ox}}}{d t}=K_{L} c_{r} c_{\mathrm{LDL}}
$$

where $k_{L}, c_{r}, c_{\mathrm{ox}}$, and $c_{\mathrm{LDL}}$ are reaction rate, radical concentration, the oxidized LDL concentration, and the average LDL concentration in intima, respectively [10]. Evolution of radical concentration is due to loss of radicals through oxidation reactions, a source term and a reduction in concentration through diffusion [10]. Reduction through diffusion is larger than oxidation reactions, so we can simplify the production of radicals as:

$$
\frac{d c_{r}}{d t}=\sigma_{r}-d_{r} c_{r}
$$

where $\sigma_{r}$ is source term, and $d_{r}$ is degradation rate [10]. Assuming constant $\sigma_{r}$ and $d_{r}$, an analytical solution can be suggested for Eq. (10):

$$
c_{r}=\frac{\sigma_{r}}{d_{r}}-c_{r 0} \operatorname{Exp}\left(-d_{r} t\right)
$$

where $c_{r 0}$ is a constant specified by initial condition. This solution consists of a transient term and a constant steady term. The transient term has a time constant given by $d_{r}$ whose order is hour. Since our calculation is based on year, we can simply eliminate transient term and define radical concentration as:

$$
c_{r}=\frac{\sigma_{r}}{d_{r}} .
$$


There is a significant and direct correlation between oxidized LDL and IMT, as reported by Savoiu [11]. The correlation shows that:

$$
\frac{d c_{\mathrm{ox}}}{d e}=25.5 \frac{\mathrm{mg} / \mathrm{dl}}{\mathrm{mm}} \text {. }
$$

Finally, the rate of intima-media thickening can be measured by Eqs. (9) to (13) as:

$$
\frac{d e}{d t}=\frac{k_{L} \frac{\sigma_{r}}{d_{r}} c_{\mathrm{LDL}}}{25.5} .
$$

All the values of the required physiological parameters for numerical and analytical solutions are provided in Table 1. Permeability, effective diffusivity and porosity of each layer are different and unique. Any change in properties of a layer can have a significant influence on transmural pressure and LDL concentration.

\section{Numerical solution for the straight tube artery}

\subsection{Geometry}

The artery is assumed as a straight axisymmetric geometry with the radius of $R=3.1 \mathrm{~mm}$. The thickness of endothelium, Intima, IEL, and media are taken as $2 \mu \mathrm{m}, 10 \mu \mathrm{m}, 2 \mu \mathrm{m}$, and $200 \mu \mathrm{m}$, respectively.

\subsection{Boundary conditions}

The boundary conditions, as shown in Figure 1, for momentum equations are: specified parabolic profile for velocity at the inlet of the arterial lumen, constant pressure at the outlet of the arterial wall, and mediaadventitia interface and zero radial velocity at the axis of symmetry. Additionally, continuity of velocity and shear stress are applied at interfaces.

\begin{tabular}{|c|c|c|c|}
\hline Layers & Parameters & Value & Ref. \\
\hline \multirow{3}{*}{ Lumen } & Density $\rho, \mathrm{g} / \mathrm{mm}^{3}$ & $1.057 \times 10^{-3}$ & [9] \\
\hline & Diffusivity $D, \mathrm{~mm}^{2} / \mathrm{s}$ & $2.87 \times 10^{-5}$ & {$[9,18]$} \\
\hline & Dynamic viscosity $\mu, \mathrm{g} /(\mathrm{mm} \mathrm{s})$ & $3.70 \times 10^{-3}$ & {$[9,18]$} \\
\hline \multirow{6}{*}{ Endothelium } & Permeability $K, \mathrm{~mm}^{2}$ & $4.32 \times 10^{-15}$ & {$[9,18,19]$} \\
\hline & Effective diffusivity $D, \mathrm{~mm}^{2} / \mathrm{s}$ & $6.00 \times 10^{-11}$ & {$[9,18,19]$} \\
\hline & Dynamic viscosity $\mu, \mathrm{g} /(\mathrm{mm} \mathrm{s})$ & $0.72 \times 10^{-3}$ & {$[9,19]$} \\
\hline & Filtration reflection coefficient $\sigma_{f}$ & 0.9979 & {$[9,19]$} \\
\hline & Osmotic reflection coefficient $\sigma_{d}$ & 0.9979 & {$[9,19]$} \\
\hline & Porosity $\varepsilon$ & 0.0005 & {$[9]$} \\
\hline \multirow{8}{*}{ Intima } & Permeability $K, \mathrm{~mm}^{2}$ & $2.00 \times 10^{-10}$ & {$[9,20]$} \\
\hline & Effective diffusivity $D, \mathrm{~mm}^{2} / \mathrm{s}$ & $5.40 \times 10^{-6}$ & {$[9,20]$} \\
\hline & Dynamic viscosity $\mu, \mathrm{g} /(\mathrm{mm} \mathrm{s})$ & $0.72 \times 10^{-3}$ & {$[9,19]$} \\
\hline & Filtration reflection coefficient $\sigma_{f}$ & 0.8272 & {$[9,19]$} \\
\hline & Porosity $\varepsilon$ & 0.983 & {$[9,20]$} \\
\hline & Oxidation reaction rate $k_{L}, \mathrm{mgs}^{-1}$ & $1 \times 10^{-4}$ & {$[10,21]$} \\
\hline & Source term $\sigma_{r}, \mathrm{mgs}^{-1}$ & $1 \times 10^{-5}$ & {$[10,21]$} \\
\hline & Degradation rate $d_{r}, \mathrm{~s}^{-1}$ & 1.39 & {$[10,21]$} \\
\hline \multirow{6}{*}{ IEL } & Permeability $K, \mathrm{~mm}^{2}$ & $4.392 \times 10^{-13}$ & {$[9,18,19]$} \\
\hline & Effective diffusivity $D, \mathrm{~mm}^{2} / \mathrm{s}$ & $3.18 \times 10^{-9}$ & {$[9,18,19]$} \\
\hline & Dynamic viscosity $\mu, \mathrm{g} /(\mathrm{mm} \mathrm{s})$ & $0.72 \times 10^{-3}$ & {$[9,19]$} \\
\hline & Filtration reflection coefficient $\sigma_{f}$ & 0.9827 & {$[9,19]$} \\
\hline & Osmotic reflection coefficient $\sigma_{d}$ & 0.9827 & {$[9,19]$} \\
\hline & Porosity $\varepsilon$ & 0.002 & {$[9]$} \\
\hline \multirow{6}{*}{ Media } & Permeability $K, \mathrm{~mm}^{2}$ & $2 \times 10^{-12}$ & {$[9,20]$} \\
\hline & Effective diffusivity $D, \mathrm{~mm}^{2} / \mathrm{s}$ & $5.00 \times 10^{-8}$ & {$[9,20]$} \\
\hline & Dynamic viscosity $\mu, \mathrm{g} /(\mathrm{mm} \mathrm{s})$ & $0.72 \times 10^{-3}$ & {$[9,20]$} \\
\hline & Filtration reflection coefficient $\sigma_{f}$ & 0.8836 & {$[9,20]$} \\
\hline & Reaction rate $k, 1 / \mathrm{s}$ & $3.197 \times 10^{-4}$ & {$[9,20]$} \\
\hline & Porosity $\varepsilon$ & 0.258 & [9] \\
\hline
\end{tabular}

Table 1. Physiological parameters. 


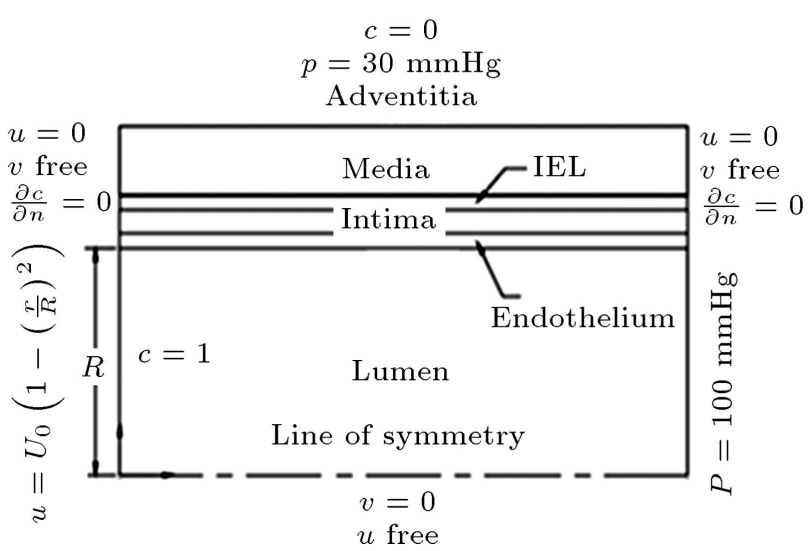

Figure 1. Schematic of the geometry of a straight tube artery.

The concentration boundary conditions are zero normal diffusive flux at the arterial wall inlet and outlet. It should be noted that the mass transport in the lumen is ignored due to its negligible effect. The concentration in media-adventitia interface is taken zero. At other interfaces, mass transport boundary condition is defined as:

$$
\left[(1-\sigma) v c-D \frac{\partial c}{\partial n}\right]_{+}=\left[(1-\sigma) v c-D \frac{\partial c}{\partial n}\right]_{-},
$$

where positive and negative signs show two sides of the interface.

\subsection{Computational approach}

The artery is assumed as a straight axisymmetric geometry. The pressure-bases finite-volume algorithm SIMPLE is employed to solve the steady flow. Twodimensional, four-noded square elements are used in the present simulation. A fine mesh is implemented near the interfaces in order to capture high LDL concentration gradients. Also, to attain grid-independent results, numerical experimentation is performed.

\section{Analytical solution for the straight tube artery}

Considering a straight pipe, as arterial wall, there are analytical solutions for Eqs. (4)-(6). LDL transport is assumed to be in radial direction, because the filtration velocity in the radial direction is far larger than the filtration velocity in the axial direction [12]. It is also reported in [9] that due to large resistance offered by arterial wall, impact of pulsation on LDL transport across the arterial wall is negligible for a straight wall [9]. Based on these assumptions, Eqs. (4)-(6) are simplified as:

Endothelium, intima and IEL:

$$
\frac{1}{\operatorname{Re} D a} v^{*}=-\frac{\partial p^{*}}{\partial y^{*}}
$$

$$
\begin{aligned}
& (1-\sigma) v^{*} C \frac{\partial c}{\partial r^{*}}=\frac{1}{\operatorname{Pe}} \frac{1}{r^{*}} \frac{\partial}{\partial r^{*}}\left(r^{*} \frac{\partial c}{\partial r^{*}}\right), \\
& \frac{\partial v^{*}}{\partial r^{*}}=0 .
\end{aligned}
$$

Media:

$$
\begin{aligned}
& \frac{1}{\operatorname{Re} D a} v^{*}=-\frac{\partial p^{*}}{\partial y^{*}} \\
& (1-\sigma) v^{*} C \frac{\partial c}{\partial r^{*}}=\frac{1}{\operatorname{Pe}} \frac{\partial^{2} c}{\partial r^{* 2}}+\frac{k R}{V_{0}} C, \\
& \frac{\partial v^{*}}{\partial r^{*}}=0
\end{aligned}
$$

Dimensionless parameters are defined as:

$$
\begin{aligned}
& v^{*}=\frac{v}{v_{0}}, \quad C=\frac{c}{c_{0}}, \quad p^{*}=\frac{p}{\rho v_{0}^{2}}, \\
& r^{*}=\frac{r}{R}, \quad Q^{*}=V^{*} r^{*}, \quad \operatorname{Re}=\frac{\rho v_{0} R}{\mu}, \\
& D a=K / R^{2}, \quad \mathrm{Pe}=\frac{v_{0} R}{D e},
\end{aligned}
$$

where $V_{0}$ is the filtration velocity obtained from numerical data and is chosen as $2.31 \times 10^{-5} \mathrm{~mm} / \mathrm{s}$, and $R$ is radius of arterial wall, which is equal to $3.1 \mathrm{~mm} ; c_{0}$ is the LDL concentration in the lumen.

The boundary conditions for momentum equations are defined as:

$$
p^{*}\left(r_{1}^{*}\right)=p_{1}^{*}, \quad p^{*}\left(r_{s}^{*}\right)=p_{2}^{*},
$$

where at first $p_{2}$ is chosen as $30 \mathrm{mmHg}$ and $p_{1}$ is chosen as $100 \mathrm{mmHg}$, then, they, respectively, are chosen as $150 \mathrm{mmHg}$ and $190 \mathrm{mmHg}$ in order to investigate hypertension effect.

The corresponding concentration boundary conditions at interfaces can be written as [12]:

- Lumen-Endothelium:

$$
C\left(r^{*}=r_{1}^{*}\right)=C_{\text {Lumen }} .
$$

- Media-Adventitia:

$$
C\left(r^{*}=r_{5}^{*}\right)=C_{\text {adventitia }} .
$$

- Endothelium-intima, intima-IEL, and IEL-media:

$$
\left[(1-\sigma) v^{*} c-\frac{1}{\operatorname{Pe}} \frac{\partial c}{\partial n}\right]_{+}=\left[(1-\sigma) v^{*} c-\frac{1}{\operatorname{Pe}} \frac{\partial c}{\partial n}\right]_{-}
$$

Assuming steady state flow, we can use hydraulic 


$$
Q=\frac{p_{1}^{*}-p_{2}^{*}}{\left(\frac{\operatorname{Ln}\left(\frac{r_{2}^{*}}{r_{1}^{*}}\right)}{\operatorname{Re} D a}\right)_{\text {end }}+\left(\frac{\operatorname{Ln}\left(\frac{r_{3}^{*}}{r_{2}}\right)}{\operatorname{Re} D a}\right)_{\mathrm{int}}+\left(\frac{\operatorname{Ln}\left(\frac{r_{4}^{*}}{r_{3}^{*}}\right)}{\operatorname{Re} D a}\right)_{\mathrm{LEL}}+\left(\frac{\operatorname{Ln}\left(\frac{r_{8}^{*}}{r_{4}^{*}}\right)}{\operatorname{Re} D a}\right)_{\mathrm{med}}}
$$

where $r_{1}^{*}, r_{2}^{*}, r_{3}^{*}, r_{4}^{*}$, and $r_{5}^{*}$ are dimensionless radial distances from lumen-endothelium, endothelium-intima, intima-IEL, and IEL-media interfaces to axis of symmetry, respectively. Subscripts "lumen", "end", "int", "IEL" and "med" refer to lumen, endothelium, intima, IEL and media, respectively.

\section{Box I}

cylindrical resistances to simplify momentum equation. The resistance is defined as:

$$
R=\operatorname{Ln}\left(\frac{r_{1}^{*}}{r_{2}^{*}}\right) /(\operatorname{Re} D a) .
$$

By use of series resistances, dimensionless flow rate can be calculated by Eq. (28) as shown in Box I.

The mass transport equations concerning endothelium, intima, and IEL can be obtained as:

$$
C\left(r^{*}\right)=c_{1}+c_{2} r^{* \lambda},
$$

where $c_{1}$ and $c_{2}$ are constant. $\lambda$ is defined as:

$$
\lambda=(1-\sigma) Q^{*} \mathrm{Pe} .
$$

The mass transport equation for media can be obtained as:

$$
\begin{aligned}
& C\left(r^{*}\right)=c_{1} e^{\lambda_{1} R^{*}}+c_{2} e^{\lambda_{2} R^{*}}, \\
& \left(\lambda_{1,2}\right)_{\text {media }} \\
& \quad=\frac{\operatorname{Pe}\left(1-\sigma^{f}\right) v^{*} \pm \sqrt{\left(\operatorname{Pe}\left(1-\sigma^{f}\right) v^{*}\right)^{2}-\frac{4 k R \mathrm{Pe}}{V_{0}}}}{2},
\end{aligned}
$$

where $c_{1}$ and $c_{2}$ are constants.

Concentration and flux of LDL (Eq. (26)) must not alter at interfaces where the layers change. Therefore, there are two types of equations which can be written as:

- Concentration in different interfaces:

$$
\begin{aligned}
& C_{\text {lumen }}=c_{1}^{\text {end }}+c_{2}^{\text {end }} r_{1}^{\lambda^{\text {end }}}, \\
& c_{1}^{\text {end }}+c_{2}^{\text {end }} r_{2}^{\lambda^{\text {end }}}=c_{1}^{\text {int }}+c_{2}^{\text {int }} r_{2}^{\lambda^{\text {int }}}, \\
& c_{1}^{\text {int }}+c_{2}^{\text {int }} r_{3}^{\lambda^{\text {int }}}=c_{1}^{\mathrm{LEL}}+c_{2}^{\mathrm{LEL}} r_{3}^{\lambda^{i \mathrm{LEL}}}, \\
& c_{1}^{\mathrm{LEL}}+c_{2}^{\mathrm{LEL}} r_{4}^{\lambda^{\text {iLEL }}}=c_{1}^{\text {med }} e^{\lambda_{1}^{\text {med }} r_{4}}+c_{2}^{\text {med }} e^{\lambda_{2}^{\text {med }} r_{4}}, \\
& c_{1}^{\text {med }} e^{\lambda_{1}^{\text {med }} r_{5}}+c_{2}^{\text {med }} e^{\lambda_{2}^{\text {med }} r_{5}}=0 .
\end{aligned}
$$

- Flux in different interfaces:

$$
\begin{aligned}
&(1\left.-\sigma^{\text {end }}\right) \frac{Q^{*}}{r_{2}}\left(c_{1}^{\text {end }}+c_{2}^{\text {end }} r_{2}^{\lambda^{\text {end }}}\right) \\
&-\frac{1}{P e^{\text {end }}} \lambda^{\text {end }} c_{2}^{\text {end }} r_{2}^{\lambda^{\text {end }}-1} \\
&=\left(1-\sigma^{\text {int }}\right) \frac{Q^{*}}{r_{2}}\left(c_{1}^{\text {int }}+c_{2}^{\text {int }} r_{2}^{\lambda^{\text {int }}}\right) \\
&-\frac{1}{P e^{\text {int }}} \lambda^{\text {int }} c_{2}^{\text {int }} r_{2}^{\lambda^{\text {int }}-1}, \\
&(1\left.-\sigma^{\text {int }}\right) \frac{Q^{*}}{r_{3}}\left(c_{1}^{\text {int }}+c_{2}^{\text {int }} r_{3}^{\lambda^{\text {int }}}\right) \\
&-\frac{1}{P e^{\text {int }}} \lambda^{\text {int }} c_{2}^{\text {int }} r_{3}^{\lambda^{\text {int }}-1} \\
&=\left(1-\sigma^{\mathrm{LEL}}\right) \frac{Q^{*}}{r_{2}}\left(c_{1}^{\mathrm{LEL}}+c_{2}^{\mathrm{LEL}} r_{3}^{\lambda^{\mathrm{LEL}}}\right) \\
&-\frac{1}{P e^{\mathrm{LEL}}} \lambda^{\mathrm{LEL}} c_{2}^{\mathrm{LEL}} r_{3}^{\lambda^{\mathrm{LEL}}-1}, \\
&(1\left.-\sigma^{\mathrm{LEL}}\right) \frac{Q^{*}}{r_{4}}\left(c_{1}^{\mathrm{LEL}}+c_{2}^{\mathrm{LEL}} r_{4}^{\lambda^{\mathrm{LEL}}}\right) \\
&-\frac{1}{P e^{\mathrm{LEL}}} \lambda^{\mathrm{LEL}} c_{2}^{\mathrm{LEL}} r_{4}^{\lambda^{\mathrm{LEL}}-1} \\
&=\left(1-\sigma^{\mathrm{med}}\right) \frac{Q^{*}}{r_{4}}\left(c_{1}^{\mathrm{Ped}} e_{1}^{\lambda_{1}^{\mathrm{med}} r_{5}}+c_{2}^{\mathrm{med}} e^{\lambda_{2}^{\mathrm{med}} r_{5}}\right) \\
&\left.\lambda_{1}^{\mathrm{med}} e^{\lambda_{1}^{\mathrm{med}} r_{5}}+c_{2}^{\mathrm{med}} \lambda_{2}^{\mathrm{med}} e^{\lambda_{2}^{\mathrm{med}} r_{5}}\right) \\
&(
\end{aligned}
$$

\section{Application to a carotid artery}

The geometry of the three dimensional model for carotid bifurcation, shown in Figure 2, was derived from Gijsen et al. [13]. A parabolic velocity profile was prescribed at the inlet in which the Re number was 


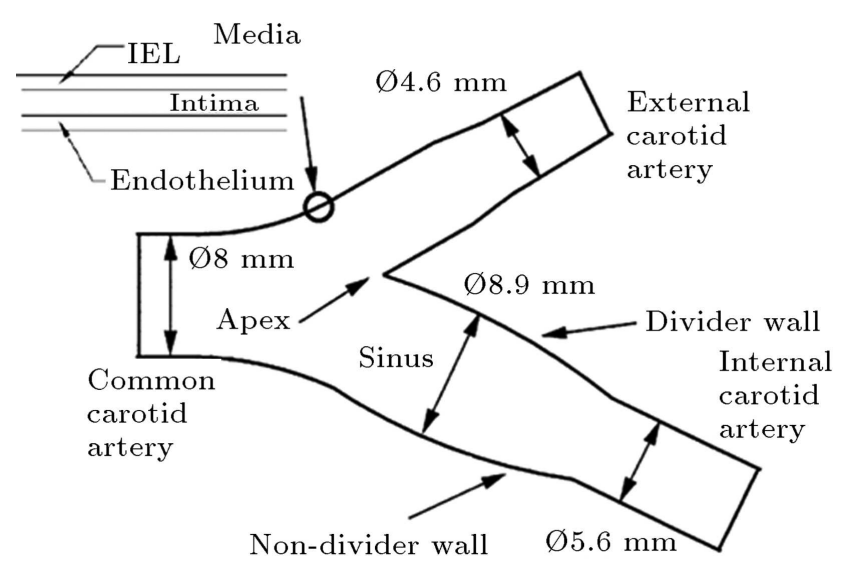

Figure 2. Schematic of the geometry of a simple carotid.

specified 270. Stress-free outflow boundary condition was used for external and internal carotid artery, and flow division ration was taken 0.45 . The wall was considered to be permeable, and the filtration velocity was obtained from Eq. (28). The concentration boundary conditions were zero diffusive flux in the flow direction at both external and internal carotid artery outlets, and constant concentration $(C=1)$ was taken at the inlet. Eqs. (33)-(40) were used as the concentration boundary condition on the permeable wall. Indeed, LDL transport in the lumen is solved numerically by use of the analytical solution for fourlayer permeable wall.

\section{Results and discussion}

\subsection{The straight tube artery}

In the present study, LDL accumulation in the arterial wall and the growth rate of the intima are investigated for various physiologically pertinent conditions. The inlet bulk flow is chosen as $U_{0}=338 \mathrm{~mm} / \mathrm{s}$ [9] and the reference concentration $c_{0}=131.7 \mathrm{mg} / \mathrm{dl}$. Transmural pressure is taken 70,120 or $160 \mathrm{mmHg}$. It is also noteworthy to mention that layer porosity inversely affects LDL uptake by changing transmural pressure. Furthermore, permeability and effective diffusivity in every layer directly influence LDL concentration and flux.

\subsubsection{LDL accumulation in the arterial wall}

It has been widely accepted that hypertension has a profound role in the development of atherosclerosis. Three factors have been reported that clarify the role of hypertension in this disease. First, an increase in transmural pressure leads to an increase in filtration velocity and mass flux. Second, an increased fluid flux causes an elevation of concentration in lumenendothelium interface. Finally, permeability may be pressure dependent [14].

Figure 3 illustrates that an increase in transmural pressure from $70 \mathrm{mmHg}$ to $160 \mathrm{mmHg}$ causes an

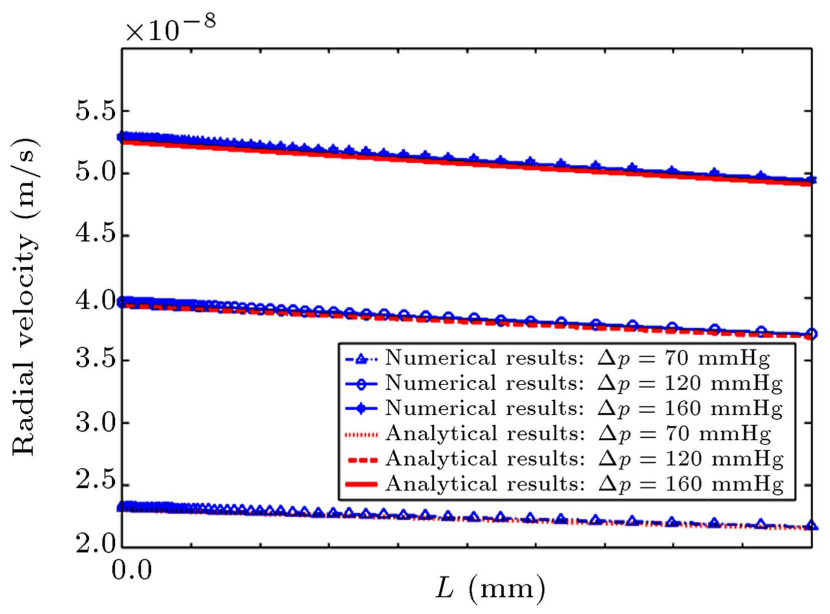

Figure 3. Computer filtration velocity across the wall based on different values of transmural pressure.

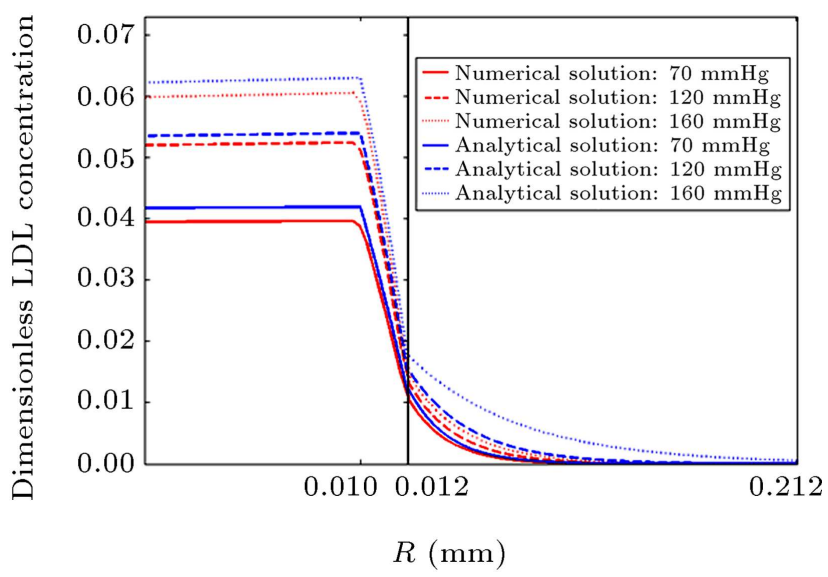

Figure 4. Computed dimensionless LDL concentration profile across different layers based on different values of transmural pressure.

increase in filtration velocity from $2.3 \times 10^{-5}$ to $5.3 \times$ $10^{-5} \mathrm{~mm} / \mathrm{s}$. It is shown that numerical solution agrees well with analytical solution.

The increased filtration velocity due to the increased transmural pressure leads to an increase in convective mass flux, and finally causes higher LDL accumulation in arterial wall, as illustrated in Figure 4. The results are in good agreement with the results of Yang and Vafai [12]. Moreover, it is also well established that LDL uptake and accumulation strongly depends on shear stress too. This fact is very crucial when studying this phenomenon locally since complex geometry of vasculature can significantly influence wall shear stress distribution.

\subsubsection{Intima growth rate}

Relying on analytical solution in different conditions such as hypertension and intimal thickness, we did not use the numerical solution which needs a large sum of computational efforts and continue the rest by employing the analytical solution. 


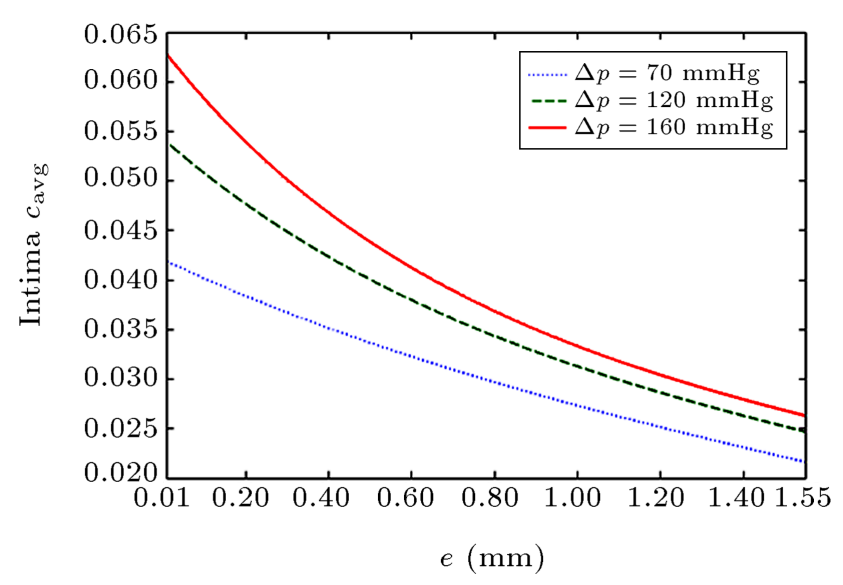

Figure 5. Computed average dimensionless LDL concentration profile across the intima based on different values of transmural pressure.

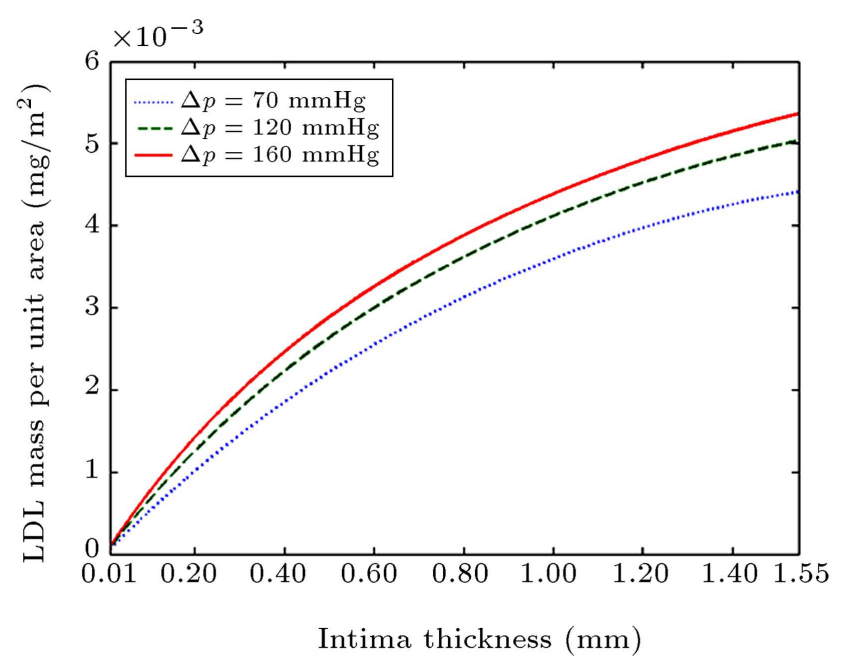

Figure 6. Computed LDL accumulation in the intima per unit area.

Figure 5 illustrates average dimensionless LDL concentration. It can be seen that an increase in transmural pressure leads to higher average concentration in intima. As shown in Figure 5, intimal thickening lowers average concentration in this layer. An increase in the arterial wall thickness results in higher hydraulic resistance, less filtration velocity, and a decrease in solute flux.

As shown in Figure 6, higher transmular pressure elevates accumulated LDL. It is also shown that rate of mass accumulation decreases when intima grows.

In order to model intimal growth rate accurately, we have to consider four stages consisting of oxidation, inflammatory process, plaque growth, and plaque instability [10]. Yet we want to have a simple model of intimal growth to focus on effects of high blood LDL concentration and hypertension.

It has been reported that a number of 74 subjects have been opted for questionnaire for identification of cardiovascular risk factors which shows that the

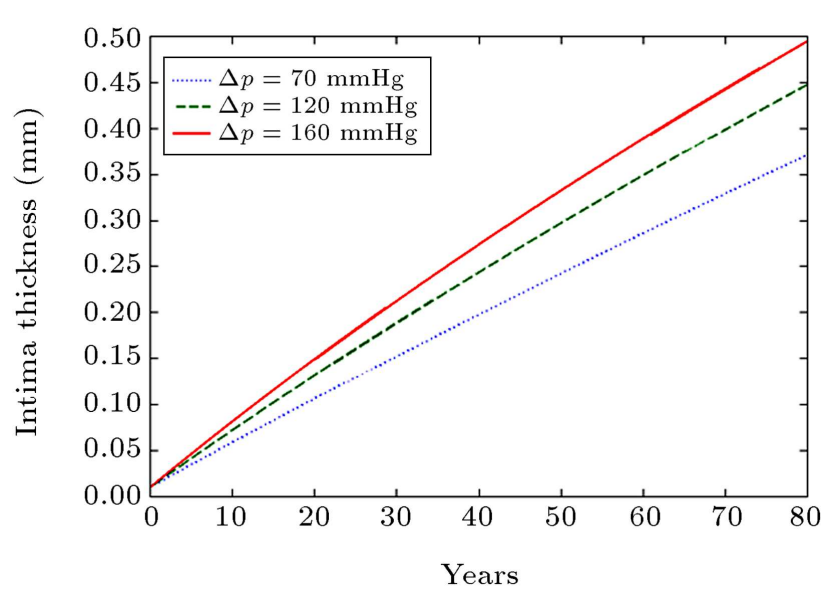

Figure 7. Computed intima thickness based on different values of transmural pressure. The average rate of intimal growth equals $4.5 \mu \mathrm{m} /$ year.

correlation between $\mathrm{LDL}_{\mathrm{Ox}}$ and $\mathrm{LDL}$ is moderate, direct, and significant [11]. Therefore, the oxidized LDL rate described in Eq. (13) is used to measure intima thickness based on the assumption that oxidation process does not change accumulated mass in the intima noticeably.

Figure 7 shows that for people having normal pressure and $131.7 \mathrm{mg} / \mathrm{dl}$ blood LDL concentration, it takes about 80 years to have $360 \mu \mathrm{m}$ increase in IMT. So, the average rate of intimal growth equals to $4.5 \mu \mathrm{m} /$ year and it match clinical reports which showed the rate of intima thickening of healthy subjects is approximately $4.5 \mu \mathrm{m} /$ year.

Gomez-Marcos et al. [15] measured carotid IMT in 121 diabetics, 352 hypertensive patients, and 89 individuals who had neither of these diseases. The mean LDL concentration of healthy subjects was $131.7 \mathrm{mg} / \mathrm{dl}$ and that is why we use that amount of concentration in our calculation. They reported that the rate of intima media growth for healthy subjects was $5 \mu \mathrm{m} /$ year which is in good agreement with our results. The rate of intimal growth in hypertensive patients is $6 \mu \mathrm{m} /$ year which shows a $20 \%$ increase. As shown in Figure 7 , our results show that the average rate of intima thickening of hypertensive patients with $120 \mathrm{mmHg}$ and $160 \mathrm{mmHg}$ transmural pressure are $5.87 \mu \mathrm{m} /$ year and $6.12 \mu \mathrm{m} /$ year, respectively.

In addition, researchers believe that the correlation between age and IMT has a linear tendency. Gomez-Marcos et al. [15] reported that the correlation coefficient for healthy subjects was 0.706 and this coefficient was measured by Homma et al. [16] as 0.84 . We have also found a linear tendency between age and IMT in this study, as well correlation coefficient was computed as 0.87 .

For every $0.1 \mathrm{~mm}$ increase in carotid IMT, the relative risk of ischemic heart disease increases by $15 \%$ [15]. Therefore, the presented model of inti- 


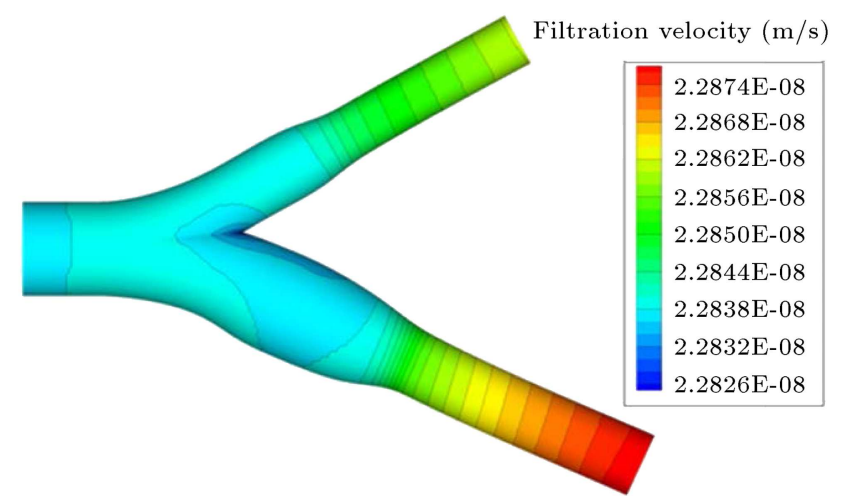

Figure 8. Filtration velocity contours across the wall for healthy subjects with $70 \mathrm{mmHg}$ tranmural pressure.

mal thickening, which can be applied to people with different transmural pressure, provides advantageous information to predict atherosclerosis susceptibility in different conditions.

\subsection{The carotid artery}

As depicted in Figure 8, the transmural velocity increases slightly along the artery. As pressure loss along the artery increases, transmural pressure increases as well, because of considering constant pressure in the media-adventitia interface. Obviously, there is not a noticeable difference between the transmural velocity obtained in the straight tube artery and the carotid including complex geometry. Indeed, both of the results of straight tube artery and the carotid artery indicate the value of $2.28 \times 10^{-8} \mathrm{~m} / \mathrm{s}$ for healthy people having normal pressure. Additionally, transmural velocity for patient people having the same carotid geometry but hypertension is obtained which has a negligible difference with the results indicated for the straight tube artery.

Dimensionless LDL concentration in the LumenEndothelium interface for a healthy subject is illustrated in Figure 9. Elevated LDL values emerge at regions opposite to the flow divider, and it ranges from 1 to 1.04 in those critical locations. This is not certainly because of transmural velocity variation, since it does not change noticeably as shown in Figure 8. As mentioned in [13], in which the geometry of the carotid is taken, an increase in the cross sectional area causes an adverse pressure gradient; therefore, the fluid is decelerated, and it affects increasingly the low impetus fluid near the non-divider wall. This could result in elevated LDL concentration in this region.

Figure 10 shows how different transmural pressure can affect LDL concentration in the LumenEndothelium interface. An increase in transmural pressure results in an increase in transmural velocity; therefore, it leads to more accumulation of LDL in the critical regions located in non-divider wall. Soulis et al.'s results [17], which agree well with ours, also show
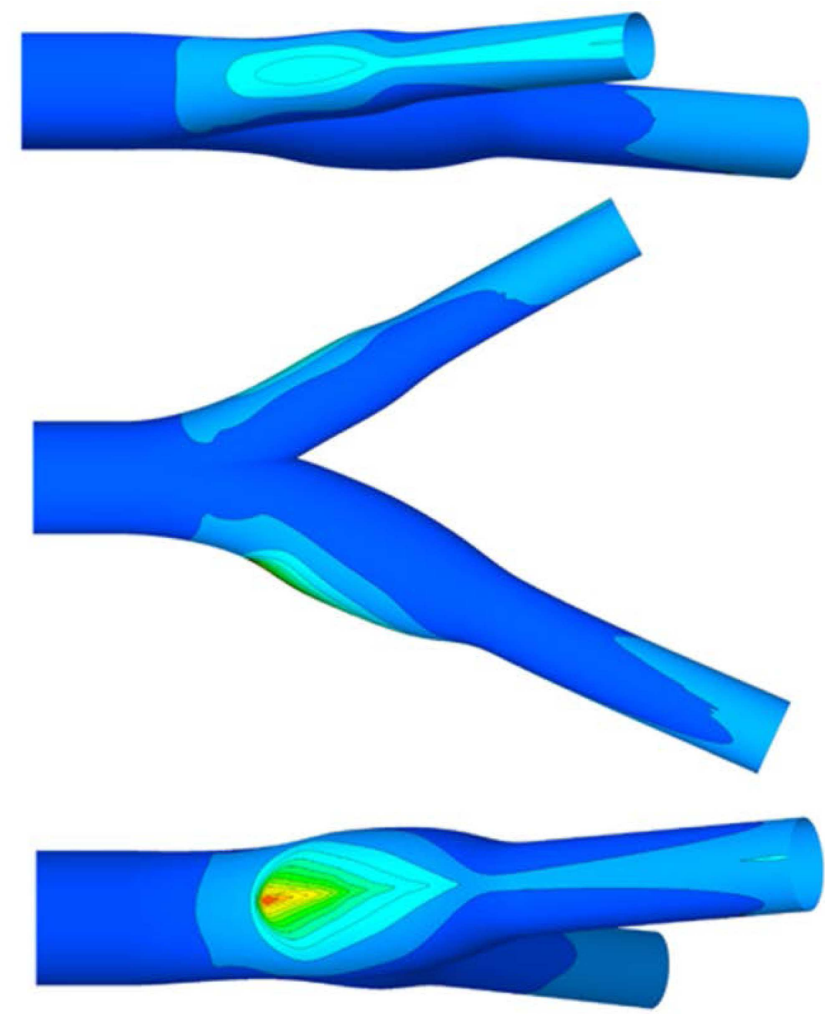

Non-dimensional LDL concentration $\left(C / C_{0}\right)$ 1.0001 .0031 .0061 .0091 .0121 .0151 .0181 .0221 .0251 .0281 .0311 .0341 .0371 .040

Figure 9. Dimensionless LDL concentration contours across the wall for healthy subjects with $70 \mathrm{mmHg}$ transmural pressure.
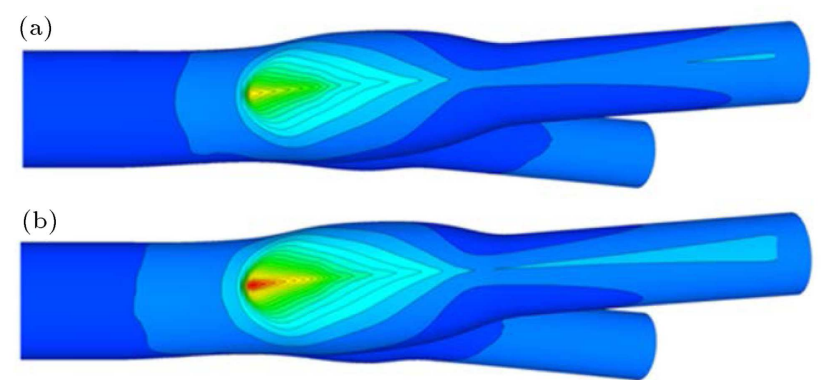

Non-dimensional LDL concentration $\left(C / C_{0}\right)$

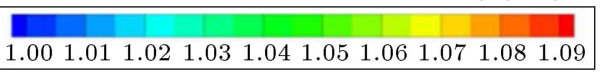

Figure 10. Dimensionless LDL concentration contours across the wall based on different transmural pressures: (a) $120 \mathrm{mmHg}$; and (b) $160 \mathrm{mmHg}$.

that an increase in transmural velocity causes elevated LDL concentration in the Lumen-Endothelium interface.

A horizontal plane, dividing the geometry into two identical parts, is used to depict mass transport more precisely. The mentioned plane has the same shape as Figure 2. LDL concentration along non- 


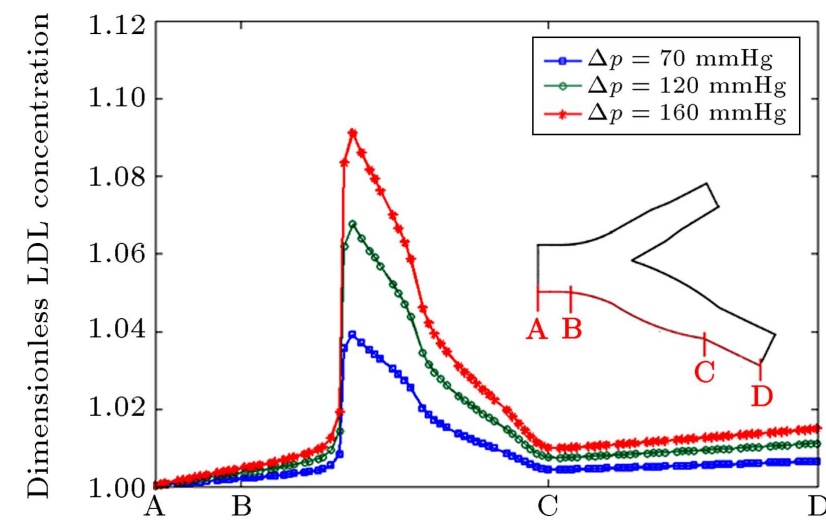

Figure 11. Dimensionless LDL concentration across the lower non-divider wall based on different transmural pressures.

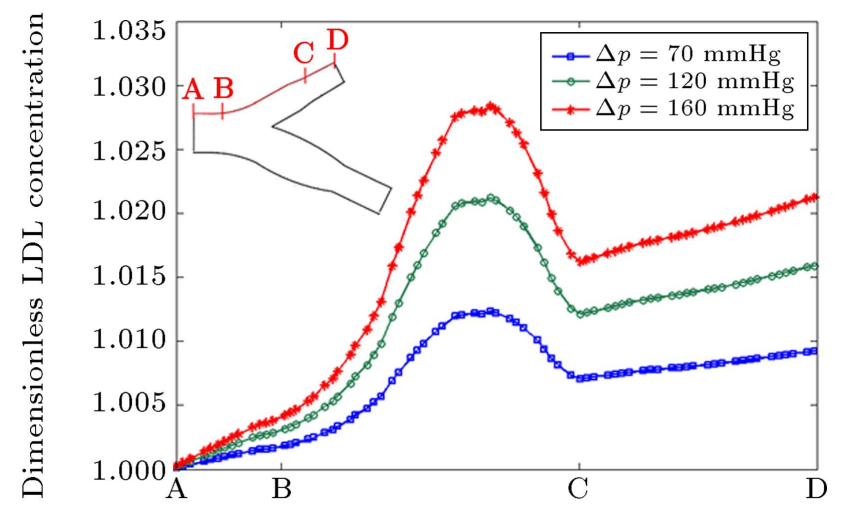

Figure 12. Dimensionless LDL concentration across the upper non-divider wall based on different transmural pressures.

divider walls are shown in Figures 11 and 12. In addition, it is graphically depicted that an increase in transmural pressure leads to a significant augmentation in LDL concentration on the wall. Also, the concentration is elevated in the region between $\mathrm{B}$ and $\mathrm{C}$ where cross section area changes and makes high curvature.

Using Eqs. (9) to (14), we obtained the intimal growth rate of the carotid artery. The maximum growth rate happens in the region between $\mathrm{B}$ and $\mathrm{C}$ on non-divider wall of internal carotid artery where the most accumulation of LDL exists. The maximum growth rate of the carotid artery for people with $70 \mathrm{mmHg}, 120 \mathrm{mmHg}$, and $160 \mathrm{mmHg}$ transmural pressure is obtained $4.68 \mu \mathrm{m} /$ year, $6.28 \mu \mathrm{m} /$ year, and $6.67 \mu \mathrm{m} /$ year, respectively.

\section{Conclusion}

Transport of LDL in the arterial wall has been studied using a homogenous porous media model. In addition, an analytical solution is presented to model LDL accumulation in the four-layer arterial wall. The proposed analytical solution has also been compared with numerical solution in different transmural pressures and different wall thicknesses.

Oxidized LDL rate is calculated using accumulated LDL in intima obtained from analytical solution. This calculated rate is a linear function of IMT. Finally, the model of intima thickening is presented which agrees well with clinical data and shows that the rate of intima thickening of healthy subjects is $4.5 \mu \mathrm{m} /$ year. Additionally, the effect of hypertension on intima accumulated LDL has been investigated. We have also calculated that the average rates of intima thickening of hypertensive patients with $120 \mathrm{mmHg}$ and $160 \mathrm{mmHg}$ transmural pressure are $4.87 \mu \mathrm{m} /$ year and $6.12 \mu \mathrm{m} /$ year, respectively.

In addition, LDL concentration on the carotid arterial wall is calculated by applying four-layer analytical solution as boundary condition to the carotid permeable wall. It is observed that LDL accumulation elevates in the non-divider wall. Then, the maximum growth rate, which is also located on the non-divider wall, is obtained by use of accumulated LDL concentration in intima.

\section{References}

1. Khakpour, M. and Vafai, K. "Critical assessment of arterial transport models", International Journal of Heat and Mass Transfer, 51, pp. 807-822 (2008).

2. Sun, N., Wood, N.B., Hughes, A.D., Thom, S.A.M. and $\mathrm{Xu}, \mathrm{X} . \mathrm{Y}$. "Fluid-wall modelling of mass transfer in an axisymmetric stenosis: effects of shear-dependent transport properties", Annals of Biomedical Engineering, 34, pp. 1119-1128 (2006).

3. Ross, R. "Atherosclerosis - An inflammatory disease", N. Engl. J. Med., 340, pp. 115-126 (1999).

4. Vafai, K., Porous Media: Applications in Biological Systems and Biotechnology, 1st Edn., Boca Raton: CRC, Taylor \& Francis, London, UK (2011).

5. Gessaghi, V.C., Raschi, M.A., Tanoni, D.Y., Perazzo, C.A. and Larreteguy, A.E. "Growth model for cholesterol accumulation in the wall of a simplified 3D geometry of the carotid bifurcation", Comput. Method Appl. Mech. Eng., 200, pp. 2117-2125 (2011).

6. Khakpour, M. and Vafai, K. "Effects of gender-related geometrical characteristics of aortailiac bifurcation on hemodynamics and macromolecule concentration distribution", International Journal of Heat and Mass Transfer, 51, pp. 5542-5551 (2008).

7. Sohrabi, S., Zheng, J., Finol, E.A. and Liu, Y. "Numerical simulation of particle transport and deposition in the pulmonary vasculature", Journal of Biomechanical Engineering, 136, 121010 (2014).

8. Sohrabi, S., Saidi, M.S., Saadatmand, M., Banazadeh, M.H. and Firoozabadi, B. "Three-dimensional simulation of urine concentrating mechanism in a functional unit of rat outer medulla. I. Model structure and base 
case results", Mathematical Biosciences, 258, pp. 44$56(2014)$.

9. Yang, N. and Vafai, K. "Modeling of low - density lipoprotein (LDL) transport in the artery-effects of hypertension", International Journal of Heat and Mass Transfer, 49, pp. 850-867 (2006).

10. McKay, C., McKee, S., Mottram, N., Mulholland, T. and Wilson, S. "Towards a model of atherosclerosis", Strathclyde Mathematics, Research Report 4 (2005).

11. Savoiu, G., Gaita, D., Fira-Mladinescu, O., et al. "Relationship of oxidized low density lipoprotein to carotid artery intima-media thickness in hypertensive patients", Bulletin UASVM, Veterinary Medicine, 65, pp. 439-443 (2008).

12. Yang, N. and Vafai, K. "Low-density lipoprotein (LDL) transport in an artery : A simplified analytical solution", International Journal of Heat and Mass Transfer, 51, pp. 497-505 (2008).

13. Gijsen, F.J.H., Vosse, F.N.v.d. and Janssen, J.D. "The influence of the non-Newtonian properties of blood on the flow in large arteries: steady flow in a carotid bifurcation model", Journal of Biomechanics, 32, pp. 601-508 (1999).

14. Ai, L. and Vafai, K. "A coupling model for macromolecule transport in a stenosed arterial wall", International Journal of Heat and Mass Transfer, 49, pp. 1568-1591 (2006).

15. Gómez-Marcosa, M.G., Recio-Rodrígueza, J.I., Rodríguez-Sáncheza, E., Patino-Alonsob, M.C., MagallónBotayac, R., Martínez-Vizcainod, V. et al. "Carotid intima-media thickness in diabetics and hypertensive patients", Revista Española de Cardiología, 64, pp. 622-625 (2011).

16. Homma, S., Hirose, N., Ishida, H., Ishii, T. and Araki, G. "Carotid plaque and intima-media thickness assessed by B-mode ultrasonography in subjects ranging from young adults to centenarians", Stroke, 32, pp. 830-835 (2001).

17. Soulis, J.V., Giannoglou, G.D., Papaioannou, V., Parcharidis, G.E. and Louridas, G.E. "Low-density lipoprotein concentration in the normal left coronary artery tree", BioMedical Engineering OnLine, 7, pp. $7-26$ (2008).

18. Karner, G., Perktold, K. and Zehentner, H.P. "Computational modeling of macromolecule transport in the arterial wall", Computer Methods in Biomechanics and Biomedical Engineering, 4, pp. 491-504 (2001).

19. Prosi, M., Address, P.Z., Perktold, K. and Quarteroni, A. "Mathematical and numerical models for transfer of low-density lipoproteins through the arterial walls: a new methodology for the model set up with applications to the study of disturbed luminal flow", Journal of Biomechanics, 38, pp. 903-917 (2005).
20. Huang, Y., Rumschitzki, D., Chien, S. and Weinbaum, S. "A fiber matrix model for the growth of macromolecular leakage spots in the arterial intima", Journal of Biomechanical Engineering, 116, pp. 430-445 (1994)

21. Cobbold, C.A., Sherratt, J.A. and Maxwell, S.R.J. "Lipoprotein oxidation and its Significance for atherosclerosis: a mathematical approach", Bulletin of Mathematical Biology, 64, pp. 65-95 (2002).

\section{Biographies}

Seyyed Amir Mirbagheri is a PhD student within the Mechanical Engineering program at University of Nevada, Reno, USA. He received his MS degree from Sharif University of Technology, Tehran, Iran, in 2012. His main areas of research interests are lowReynolds number hydrodynamics and solid and fluid mechanics of swimming microorganisms and microengineered systems.

Mohammad Said Saidi is Professor of Mechanical Engineering at Sharif University of Technology, Tehran, Iran. His research interests include: modeling and numerical analysis of transport and deposition of aerosol particles, modeling and numerical analysis of biofluids, modeling and numerical analysis of thermalhydraulics in porous media, and microchannels.

Salman Sohrabi is a PhD student within the Mechanical Engineering program at Lehigh University, USA. He received his MS degree from Sharif University of Technology, Tehran, Iran, in 2013. His main areas of research interests are fluid dynamics, drug delivery, micro/nano engineering for biology and medicine, in particular, using combined computational and experimental fluid/solid mechanics approaches to characterize the interfacial phenomena at the micro/nano scale.

Bahar Firoozabadi His/her biography was not available at the time of publication.

Mohamad Hossein Banazadeh is a PhD student within the Mechanical Engineering program at University of Maryland, USA. He received his MS degree from Sharif University of Technology, Tehran, Iran, in 2013. His main areas of research interests are microfluidic systems, multi-organ fluidic devices for in vitro assessment and evaluation of a novel therapeutic regimen for leukemia chemotherapy, multiphase flow and particle tracking, bio-fluid mechanics, and fluid flow and transport phenomena in bio-organs (especially in the kidney functional unit). 